TRANSACTIONS OF THE

AMERICAN MATHEMATICAL SOCIETY

Volume 179, May 1973

\title{
CONCERNING THE SHAPES OF FINITE-DIMENSIONAL COMPACTA
}

BY

\author{
ROSS GEOGHEGAN(1) AND R. RICHARD SUMMERHILL
}

\begin{abstract}
It is shown that two "tamely" embedded compacta of dimension $\leq k$ lying in $E^{n}(n \geq 2 k+2)$ have the same (Borsuk) shape if and only if their complements are homeomorphic. In particular, two $k$-dimensional closed submanifolds of $E^{2 k+2}$ have the same homotopy type if and only if their complements are homeomorphic.
\end{abstract}

1. Introduction. We characterize the shapes (in the sense of Borsuk) of compact subsets of a euclidean space in terms of the homeomorphism types of their complements. The characterization is stated in its basic form (Theorem 1.1) and then in a more useful form (Theorem 1.2).

But first some definitions. A closed subset $X$ of a topological space $M$ is a $Z_{k}$ set $(k$ an integer $\geq 0)$ if for every nonempty $k$-connected open set $U$ in $M$, $U-X$ is nonempty and $k$-connected. If $M$ is a metric space and $\varepsilon>0$, we denote the set of points whose distance from $X$ is less than $\varepsilon$ by $N(X, \varepsilon)$. An $\varepsilon$ push $h$ of the pair $\left(E^{n}, X\right)$ is a homeomorphism of the $n$-dimensional euclidean space $E^{n}$ for which an $\varepsilon$-isotopy $H$ of $E^{n}$ exists satisfying: $H_{0}=1, H_{1}=h$ and $H_{t}$ fixes the complement of $N(X, \varepsilon)$ for each $t$ in the unit interval $I$. A closed subset $X$ of $E^{n}$ is a strong $Z_{k}$-set $(k \geq 0)$ if for each compact subpolyhedron $P$ of $E^{n}$ having dimension $\leq k+1$, and each $\varepsilon>0$, there exists an $\varepsilon$-push $h$ of $\left(E^{n}, X\right)$ such that $h(X) \cap P=\varnothing$. For the definition of the shape of a compactum $X$, we refer to [4]. As is customary, the notation $\operatorname{Sh}(X)=\operatorname{Sh}(Y)$ means that $X$ and $Y$ have the same shape.

We can now state our main theorems.

Theorem 1.1. Let $X$ and $Y$ be nonempty compact strong $Z_{n-k-2}$-sets in $E^{n}$ $(k \geq 0, n \geq 2 k+2)$. Then the following are equivalent:

(1) $\operatorname{Sh}(X)=\operatorname{Sh}(Y)$;

(2) $\left(E^{n} / X, \hat{X}\right)$ and $\left(E^{n} / Y, \hat{Y}\right)$ are homeomorphic as pairs;

(3) $\left(E^{n} / X, E^{n} / X-\hat{X}, \hat{X}\right)$ and $\left(E^{n} / Y, E^{n} / Y-\hat{Y}, \hat{Y}\right)$ have the same homotopy type as triples;

(4) $E^{n}-X$ and $E^{n}-Y$ are homeomorphic.

$\hat{X}$ and $\hat{Y}$ denote the quotient points of the quotient spaces $E^{n} / X$ and $E^{n} / Y$. The meaning of "homotopy type of triples" might be guessed, but it is given in $\S 4$ before Lemma 4.3 .

Presented to the Society, December 20, 1971; received by the editors January 3, 1972 and, in revised form, June 20, 1972.

AMS (MOS) subject classifications (1970). Primary 57Al5; Secondary 55D10, 57A30, 58B05.

(1) Supported in part by National Science Foundation grant GP $7952 X 3$. 
The property "strong $Z_{n-k-2}$ " is not a homotopy property and so it cannot be easily verified in practice. In $\$ 2$ we review the literature of taming theorems, and show that certain homotopy properties often imply the property strong $Z_{n-k-2}$. That work enables us to prove

Theorem 1.2. Let $X$ and $Y$ be nonempty compact subsets of $E^{n}$ and let either of the following conditions hold:

(a) $E^{n}-X$ and $E^{n}-Y$ are $1-U L C, n \neq 4, n \geq \max (2 \operatorname{dim} X+2,2 \operatorname{dim} Y$ $+2)$, or

(b) $X$ and $Y$ are $Z_{n-k-2}$-sets, $k \geq 0, n \geq 2 k+2, n \neq 4$.

Then the following are equivalent:

(1) $\operatorname{Sh}(X)=\operatorname{Sh}(Y)$;

(2) $\left(E^{n} / X, \hat{X}\right)$ and $\left(E^{n} / Y, \hat{Y}\right)$ are homeomorphic as pairs;

(3) $\left(E^{n} / X, E^{n} / X-\hat{X}, \hat{X}\right)$ and $\left(E^{n} / Y, E^{n} / Y-\hat{Y}, \hat{Y}\right)$ have the same homotopy type as triples;

(4) $E^{n}-X$ and $E^{n}-Y$ are homeomorphic.

As usual 1-ULC means uniformly locally simply connected. Note that there is no explicit dimension restriction in (b) of Theorem 1.2.

Theorem 1.1 suggests the question: Which compacta can be embedded in $E^{n}$ as strong $Z_{n-k-2}$-sets? The answer is given by

Proposition 1.3. Every compactum of dimension $\leq k$ can be embedded in $E^{n}$ $(n \geq 2 k+1)$ as a strong $Z_{n-k-2}$-set.

It is time to admit that ours is not the first characterization of shapes of finitedimensional compacta in terms of homeomorphic complements. The first such theorem (that we know of) is due to Chapman [9] and can be stated as follows:

Let $X$ and $Y$ be compacta of dimension $\leq k$; (i) there exist copies $X^{\prime}$ (of $X$ ) and $Y^{\prime}($ of $Y)$ in $E^{n}(n \geq 2 k+2)$ such that if $\operatorname{Sh}(X)=\operatorname{Sh}(Y)$ then $E^{n}-X$ and $E^{n}-Y$ are homeomorphic; (ii) there exist copies $X^{\prime}$ (of $X$ ) and $Y^{\prime}$ (of $Y$ ) in $E^{n}$ $(n \geq 3 k+3)$ such that if $E^{n}-X^{\prime}$ and $E^{n}-Y^{\prime}$ are homeomorphic then $\operatorname{Sh}(X)$ $=\operatorname{Sh}(Y)$.

Theorems 1.1 and 1.2 together with Proposition 1.3 improve Chapman's theorem in two ways: by reducing the unnecessary condition $n \geq 3 k+3$ of (ii) to the more likely $n \geq 2 k+2$; and (more importantly) by making explicit, in elementary terms, which copies $X^{\prime}$ and $Y^{\prime}$ in $E^{n}$ are acceptable.

It is worth pointing out that in the infinite-dimensional case, Chapman has given, in [8], a characterization theorem every bit as strong as our Theorem 1.2, namely:

If $X$ and $Y$ are Z-sets in the Hilbert cube $I^{\infty}$, then $\operatorname{Sh}(X)=\operatorname{Sh}(Y)$ if and only if $I^{\infty}-X$ and $I^{\infty}-Y$ are homeomorphic.

(" $Z$-set" means " $Z_{k}$-set for all $k \geq 0$ ": a concept first introduced by Anderson in [1] and frequently used in infinite-dimensional topology.) So it would seem 
that there are analogies to be found between the point-set topology of euclidean spaces and the Hilbert cube. In another paper [10] we will show just how strong the analogies are, by defining pseudo-boundaries and pseudo-interiors in euclidean spaces, and by showing that many compact subsets of a pseudo-interior (there are several pseudo-interiors) are negligible. However, no knowledge of infinite-dimensional topology is needed for the present paper.

We also draw the reader's attention to Henderson's paper [12]. Many interesting examples are discussed there, and Theorem 1.6 of that paper appears to be related to the $(1) \Rightarrow(3)$ part of our Theorem 1.2 (for the special case $Y=$ a point).

Theorems 1.1 and 1.2 are proved at the end of $\S 4$. Proposition 1.3 is simply a weak form of Corollary 3.6.

In $\$ 2$ we study the relationship between 1-ULC, $Z_{k}$ and strong $Z_{k}$. In $\$ 3$ we study a special kind of strong $Z_{k}$-set in $E^{n}$ which we call "pseudo-polyhedral": this may have independent interest (see Theorem 3.1) but, for us, it is the means by which we relate the property strong $Z_{k}$ to shape. Shape itself is only discussed in $\S 4$.

This paper can be read independently of [9].

2. Taming theorems. In this section we do the following:

(a) we prove a taming theorem (2.5) for compact strong $Z_{n-k-2}$-sets in $E^{n}$ $(n \geq 2 k+2)$

(b) we give conditions under which 1 -ULC together with dimension $\leq k$ implies strong $Z_{n-k-2}$ (Theorem 2.2);

(c) we give conditions under which $Z_{n-k-2}$ implies strong $Z_{n-k-2}$ (Theorem 2.4); and hence

(d) we state when 1-ULC sets and $Z_{n-k-2}$-sets in $E^{n}$ are tame (Corollary 2.7). In fact, we prove no new taming theorems. We merely recover the theorems of Bryant [5], [6], Štan'ko [16], Bryant-Seebeck [7], Bing-Kister [2] and McMillan [15] (using some of their crucial lemmas). But we believe there is insight to be gained by organizing things in this manner.

A metric space $M$ is $k$-ULC $(k \geq 0)$ if for each $\varepsilon>0$ there exists $\delta>0$ such that any map of the $k$-sphere $S^{k}$ into $M$ whose image has diameter $<\delta$ extends to a map of the $(k+1)$-ball $B^{k+1}$ into $M$ whose image has diameter $<\varepsilon . M$ is said to be $\mathrm{ULC}^{k}$ if $M$ is $i$-ULC for each $i=0, \ldots, k$.

The relationship between $Z_{k}$ and $U_{L C}{ }^{k}$ is given in the following proposition. We give only an outline of the elementary proof.

Proposition 2.1. Let $X$ be a compact subset of $E^{n}$ and let $k$ be a nonnegative integer. Then the following are equivalent:

(1) $X$ is a $Z_{k}$-set;

(2) $\operatorname{dim} X<n$ and $E^{n}-X$ is $U L C^{k}$; and

(3) for each $\varepsilon>0$ and each compact subpolyhedron $P$ of $E^{n}$ having dimension 
$\leq k+1$, there exists a map $f: P \rightarrow E^{n}$ such that $f(P) \cap X=\varnothing$ and $d(f(x), x)$ $<\varepsilon$ for each $x \in P$.

Proof. (1) $\Rightarrow$ (2). If $U$ is a nonempty $k$-connected open subset of $E^{n}$, then $U-X$ is nonempty and hence $X$ cannot contain an open subset; i.e., $\operatorname{dim} X<n$. Furthermore, $X$ can be covered by finitely many open sets $U_{1}, \ldots, U_{m}$ such that $U_{i}-X$ is nonempty and $k$-connected for each $i=1, \ldots, m$. By using a typical compactness argument it is easy to see that $E^{n}-X$ is $\mathrm{ULC}^{k}$.

$(2) \Rightarrow(3)$. Triangulate $P$ so that the simplexes have small diameter and then, using the fact that $\operatorname{dim} X<n$, define a map $f$ on the 0 -skeleton of this triangulation with range missing $X$. The $\mathrm{ULC}^{k}$ property can then be used to inductively extend $f$ over all the various skeleta.

(3) $\Rightarrow(1)$. Obvious.

Theorem 2.2. Let $X$ be a nonempty compact subset of $E^{n}$ having dimension $\leq k$ $(n \neq 4, n-k-2 \geq 1)$ and let $E^{n}-X$ be $1-U L C$. Then $X$ is a strong $Z_{n-k-2^{-s e t}}$

Proof. Suppose first that $k=0$. Then it follows from the discussion on p. 707 of [15] that for each $\varepsilon>0$ there is a finite, disjoint collection of PL $n$-cells, each of diameter less than $\varepsilon$, whose interiors cover $X$. But if $P$ is a compact subpolyhedron of $E^{n}$ having dimension $\leq n-1$, then there is a small push $h$ of $\left(E^{n}, X\right)$ which moves the union of the PL $n$-cells, and hence $X$, off $P$. This implies that $X$ is a strong $Z_{n-2}$-set.

Now suppose that $k=1$. If $\operatorname{dim} X=0$, then the preceding paragraph applies. We therefore assume that $X$ is 1-dimensional and we let $P$ be a compact subpolyhedron of $E^{n}$ of dimension $\leq n-2$. Let $g: X \rightarrow E^{n}$ be a map such that $g(X)$ is a 1-dimensional compact subpolyhedron of $E^{n}$ and which moves points a short distance (see [14, Chapter 5]). By general position we may assume that $g(X) \cap P=\varnothing$. Since $n \geq 5$ for this case, we may approximate $g$ by an embedding $f$ such that $f(X) \cap P=\varnothing$. Then applying Lemma 2 of [5] when $n \geq 6$ or Lemma 3 of [6] when $n=5$, there is a small push $h$ of $\left(E^{n}, X\right)$ such that $h \mid X$ is close enough to $f$ to ensure $h(X) \cap P=\varnothing$. Hence $X$ is a strong $Z_{n-3}$-set.

The remaining case is $k \geq 2$. If this holds, then $n-k-1 \leq n-3$ and Lemma 1 of [5] implies directly that $X$ is a strong $Z_{n-k-2}$-set.

Lemma 2.3. Let $X$ be a compact strong $Z_{n-k-2}$-set in $E^{n}$. Then $\operatorname{dim} X \leq k$.

Proof. Let $N$ be a small polyhedral neighborhood of $X$ in $E^{n}$ which is equipped with a triangulation of small mesh. Let $N^{k}$ be the body of the $k$-skeleton and $\tilde{N}^{n-k-1}$ the body of the dual $(n-k-1)$-skeleton. Since $X$ is a strong $Z_{n-k-2}$-set, we can push $X$ off $\tilde{N}^{n-k-1}$ and, since $\tilde{N}^{n-k-1}$ and $N^{k}$ are "complementary" skeleta, we can then push $X$ arbitrarily close to $N^{k}$. This implies $\operatorname{dim} X \leq k$ (see p. 72 of [14]). 
Theorem 2.4. Let $X$ be a nonempty compact $Z_{n-k-2}$-set in $E^{n}(k \geq 0, n-k-2$ $\geq 1, n \neq 4)$. Then $X$ is a strong $Z_{n-k-2}$-set in $E^{n}$.

Proof. First suppose that $k \geq 2$. By Proposition 2.1, $E^{n}-X$ is $\mathrm{ULC}^{n-k-2}$. The theorem therefore follows from the proof of Lemma 1 of [5], since $n-k-1$ $\leq n-3$ and the essential property used in that proof is that $E^{n}-X$ is ULC $^{n-k-2}$. Now suppose that $k<2$ and consider $X \times I^{2} \subset E^{n} \times E^{2}=E^{n+2}$. It is easily seen that $X \times I^{2}$ is a $Z_{(n+2)-(k+2)-2}$-set in $E^{n+2}$ and hence, by the first case we considered, a strong $Z_{n-k-2}$-set in $E^{n+2}$. By 2.3, this implies that $\operatorname{dim}\left(X \times I^{2}\right)$ $\leq k+2$. But then $\operatorname{dim} X \leq k$ by the Remark on p. 34 of [14] and the theorem now follows from 2.2 .

Theorem 2.5 (Taming theorem). Let $X$ and $Y$ be nonempty compact strong $Z_{n-k-2}$-sets in $E^{n}(k \geq 0, n \geq 2 k+2)$ and let $f: X \rightarrow Y$ be a homeomorphism such that $d(x, f(x))<\varepsilon$ for each $x$ in $X$. Then there is an $\varepsilon$-push $h$ of $\left(E^{n}, X\right)$ such that $h \mid X=f$.

Theorem 2.5 is proved by combining the techniques of the proof of Theorem 4.4 of [11] with the following.

Lemma 2.6. Let $X$ be a nonempty compact strong $Z_{n-k-2}$-set $X$ in $E^{n}$ $(k \geq 0, n \geq 2 k+2)$ and let $f: X \rightarrow E^{n}$ be an embedding such that $d(x, f(x))<\varepsilon$ for each $x$ in $X$. Then for each $\delta>0$, there is an $\varepsilon$-push $h$ of $\left(E^{n}, X\right)$ such that $d(f(x), h(x))<\delta$ for each $x$ in $X$.

Proof. We shall give only an outline since the proof can be found on pp. 48-49 of [5]. First extend $f$ to a map from $E^{n}$ to $E^{n}$ and then push $X$ close to $N^{k}$, where $N^{k}$ is as in the proof of 2.3. But now a taming theorem is known for polyhedra in the trivial range [2] and hence $N^{k}$ can be pushed close to $f\left(N^{k}\right)$. This last push then drags $X$ along with $N^{k}$ and thus $X$ is pushed close to $f(X)$.

Corollary 2.7. Let $X$ and $Y$ be nonempty compact subsets of $E^{n}$ and let either of the following conditions hold:

(1) $E^{n}-X$ and $E^{n}-Y$ are $1-U L C, n \neq 4, n \geq 2 \operatorname{dim} X+2$; or

(2) $X$ and $Y$ are $Z_{n-k-2}$-sets, $k \geq 0, n \geq 2 k+2, n \neq 4$.

Then if $f: X \rightarrow Y$ is a homeomorphism such that $d(x, f(x))<\varepsilon$ for each $x$ in $X$, there is an $\varepsilon$-push $h$ of $\left(E^{n}, X\right)$ such that $h \mid X=f$.

Proof. Case (2) follows directly from Theorems 2.4 and 2.5 except when $(n, k)=(2,0)$. It is easy to show, however, that a $Z_{0}$-set in $E^{2}$ is a strong $Z_{0}$-set. In proving case (1), we may assume $(n-\operatorname{dim} X-2) \geq 1$ : this could only fail to be true when $n=2$ and $\operatorname{dim} X=0$, but then $E^{n}-X$ could not be 1-ULC. Hence case (1) follows from Theorems 2.2 and 2.5. This completes the proof.

We conclude this section with slightly different versions of 2.5 and 2.7 which will be useful in $\$ 4$. 
Theorem 2.8. Let $X$ and $Y$ be nonempty compact strong $Z_{n-k-2}$-sets in $E^{n}$ $(k \geq 0, n \geq 2 k+2)$ and let $f: X \rightarrow Y$ be a homeomorphism which is homotopic to $1_{X}$ in an open neighborhood $U$ of $X \cup Y$. Then $f$ extends to a homeomorphism $h$ of $E^{n}$ such that $h$ fixes the complement of $U$.

Proof. This follows from 2.5, 2.6 and 2.3. One simply moves along the homotopy in a finite number of stages, using 2.6 and 2.3 to approximate at each stage by an embedding of $X$ whose image is a strong $Z_{n-k-2}$-set.

Corollary 2.9. Let $X$ and $Y$ be nonempty compact subsets of $E^{n}$ and let either of the following conditions hold:

(1) $E^{n}-X$ and $E^{n}-Y$ are $1-U L C ; n \neq 4, n \geq 2 \operatorname{dim} X+2$; or

(2) $X$ and $Y$ are $Z_{n-k-2}$-sets, $k \geq 0, n \geq 2 k+2, n \neq 4$.

Then if $f: X \rightarrow Y$ is a homeomorphism which is homotopic to $1_{X}$ in an open neighborhood $U$ of $X \cup Y$, f extends to a homeomorphism $h$ of $E^{n}$ such that $h$ fixes the complement of $U$.

Proof. Combine 2.2, 2.4 and 2.8.

3. Embeddings of compacta in $E^{n}$. Let $P$ be a compact subpolyhedron of $E^{n}$ and let $\varepsilon$ be a positive real number. An $\varepsilon$-regular neighborhood of $P$ in $E^{n}$ is a regular neighborhood $N$ of $P$ such that for any compact subset $Y$ of $E^{n}-P$, there is an $\varepsilon$-push $h$ of $\left(E^{n}, P\right)$ such that $h(Y) \cap N=\varnothing$. It follows trivially from regular neighborhood theory (e.g., see [13]) that $\varepsilon$-regular neighborhoods always exist for any polyhedron $P$ and any $\varepsilon>0$.

Definition 3.0. Let $X$ be a compact subset of $E^{n}$. The statement that $X$ is a pseudopolyhedral subset of $E^{n}$ means that there is a pair of sequences $\left\{P_{i}\right\},\left\{N_{i}\right\}$, $i=1,2, \ldots$, with the following properties:

(1) each $P_{i}$ is a compact subpolyhedron of $E^{n}$,

(2) $\operatorname{dim} P_{i} \leq \operatorname{dim} X$ for each $i=1,2, \ldots$,

(3) each $N_{i}$ is a (1/i)-regular neighborhood of $P_{i}$, and

(4) $X \subset$ int $N_{i} \subset N_{i} \subset N(X, 1 / i)$ for each $i=1,2, \ldots$

If $X$ is an arbitrary compactum, a pseudo-polyhedral embedding $h$ of $X$ into $E^{n}$ is an embedding such that $h(X)$ is pseudo-polyhedral.

Note. Any compact subpolyhedron of $E^{n}$ is a pseudo-polyhedral set and any PL embedding is a pseudo-polyhedral embedding.

Note. The property "pseudo-polyhedral" is more restrictive than the property "standard position" used in [9]. See [17] for an example.

In this section we discuss some properties of pseudo-polyhedral sets for use in §4. One feature of this section which may have independent interest is the following theorem (proved by combining 2.3, 2.8, 3.2, 3.3, and Theorem 1.1 of [11]): 
Theorem 3.1. The strong $Z_{n-k-2}$-sets in $E^{n}(k \geq 0, n \geq 2 k+2)$ are precisely the images of pseudo-polyhedral sets of dimension $\leq k$ under self-homeomorphisms of $E^{n}$.

Proposition 3.2. If $X$ is a pseudo-polyhedral set in $E^{n}$ having dimension $\leq k$ $(n-k-2 \geq 0)$, then $X$ is a strong $Z_{n-k-2}$-set in $E^{n}$.

Proof. We refer to Definition 3.0. By general position, a compact subpolyhedron of $E^{n}$ having dimension $\leq n-k-1$ can be pushed off any one of the polyhedra $P_{i}$ and so by (3) can be pushed off $N_{i}$. This pushes $P$ off $X$; hence $X$ can be pushed off $P$.

Theorem 3.3. Let $X$ be a compactum having dimension $\leq k$ and let $n \geq 2 k+1$. Then the collection of all pseudo-polyhedral embeddings of $X$ into $E^{n}$ is a dense $G_{\delta^{-}}$ subset of the collection of all mappings from $X$ into $E^{n}$.

The proof of this theorem will require two lemmas. We note here that the collection of all maps from $X$ into $E^{n}$, which we denote by $F[X]$, is assumed to have the uniform topology. Let $I[X]$ denote the embeddings of $X$ into $E^{n}$, let $F_{p}[X]$ denote those $f$ in $F[X]$ for which $f(X)$ is pseudo-polyhedral and $\operatorname{dim} f(X)$ $\leq k$, and let $I_{p}[X]=F_{p}[X] \cap I[X]$. If $\varepsilon>0$, an $\varepsilon$-mapping $f$ of $X$ into $E^{n}$ is a map such that $\operatorname{diam} f^{-1}(y)<\varepsilon$ for each $y \in E^{n}$. For $i=1,2, \ldots$, let $G_{i}$ denote the set of all $(1 / i)$-mappings in $F[X]$. Then the following lemma is proved on $\mathrm{p}$. 57 of [14].

Lemma 3.4. Each $G_{i}$ is a dense open subset of $F[X]$ and hence $I[X]=\bigcap_{i=1}^{\infty} G_{i}$ is a dense $G_{\delta}$-subset of $F[X]$.

For each $i=1,2, \ldots$, let $C_{i}$ denote the set of all $f$ in $F[X]$ for which there is a compact subpolyhedron $P$ in $E^{n}, \operatorname{dim} P \leq k$, and a $(1 / i)$-regular neighborhood $N$ of $P$ such that $f(X) \subset$ int $N \subset N \subset N(f(X), 1 / i)$. Note that $F_{p}[X]=\bigcap_{i=1}^{\infty} C_{i}$.

Lemma 3.5. Each $C_{i}$ is a dense open subset of $F[X]$.

Proof. If $f \in F[X]$, then arbitrarily close to $f$ there is a map $g \in F[X]$ whose image is a subpolyhedron of $E^{n}$ having dimension $\leq k$. Setting $P=g(X)$ and $N$ equal to any $(1 / i)$-regular neighborhood of $P$, we see that $g \in C_{i}$. To show $C_{i}$ is open, let $f \in C_{i}$ and let $P$ and $N$ be as in the definition of $C_{i}$. Then if $g$ is close enough to $f$ (we leave the calculation of $\varepsilon$ to the reader), $g(X) \subset$ int $N \subset N$ $\subset N(g(X), 1 / i)$.

Proof of Theorem 3.3. By Lemmas 3.4 and 3.5, $C_{i} \cap G_{i}$ is dense and open in $F[X]$ and, by Baire's theorem,

$$
\bigcap_{i=1}^{\infty}\left(C_{i} \cap G_{i}\right)=\left(\bigcap_{i=1}^{\infty} C_{i}\right) \cap\left(\bigcap_{i=1}^{\infty} G_{i}\right)=F_{p}[X] \cap I[X]=I_{p}[X]
$$


is the dense $G_{\delta}$-subset of $F[X]$.

Corollary 3.6. Let $X$ be a compactum having dimension $\leq k$ and let $n \geq 2 k+1$. Then every map of $X$ into $E^{n}$ can be uniformly approximated by an embedding whose image is a strong $Z_{n-k-2}$-set in $E^{n}$.

4. Shape. We refer the reader to [3] and [4] for definitions concerning the concepts of shape, fundamental sequences, etc. These concepts are defined for compact subsets of a particular absolute retract $W$ (e.g. separable Hilbert space). However Theorem 2.4 of [4] implies that the definitions are really independent of $W$ and of the embeddings in $W$. This is very useful for us: all our compacta will lie in a euclidean space $E^{n}$, and this theorem will allow us to define our fundamental sequences relative to $E^{n}$.

In this section, we prove Theorem 1.1 and the necessary supporting lemmas. Theorem 1.2 then follows immediately by combining Theorem 1.1 with the theorems of $\S 2$. Lemmas 4.1 and 4.2 give the $(1) \Rightarrow(2)$ part of 1.1 ; Lemma 4.3 is the main step in proving (3) $\Rightarrow(1)$; and Lemma 4.4 is used in deducing (4) $\Rightarrow$ (1) from $(2) \Rightarrow(3) \Rightarrow(1)$. We end the section with formal proofs of 1.1 and 1.2 in which we combine the lemmas of this section with those of $\$ 2$ and $\S 3$.

The proofs of our next two Lemmas 4.1 and 4.2 are modeled on Henderson's proofs of 4.1 and 1.4 in [12]. Lemma 4.1 is the inductive step in Lemma 4.2.

Lemma 4.1. Let $A$ and $B$ be nonempty compact strong $Z_{n-k-2}$-sets in $E^{n}$ $(k \geq 0, n \geq 2 k+2)$ such that $\operatorname{Sh}(A)=\operatorname{Sh}(B)$. Let $\left\{f_{i}, A, B\right\}$ and $\left\{f_{i}^{\prime}, B, A\right\}$ be fundamental sequences (in $E^{n}$ ) which are homotopy inverse to one another. Let $U_{0}$ be a neighborhood of $A$ and let $h$ be a homeomorphism of $E^{n}$ such that $B \subset h\left(U_{0}\right)$ and $h^{-1} \mid B$ is homotopic to $f_{i}^{\prime} \mid B$ in $U_{0}$ for almost all $i$. Then for every neighborhood $V_{0}$ such that $B \subset V_{0} \subset h\left(U_{0}\right)$, there is a homeomorphism $q$ of $E^{n}$ such that $q=h$ outside $U_{0}, A \subset q^{-1}\left(V_{0}\right)$ and $q \mid A$ is homotopic to $f_{i} \mid A$ in $V_{0}$ for almost all $i$.

Proof.(2) It is not difficult to choose $V$ an open neighborhood of $B$ in $V_{0}, U$ an open neighborhood of $A$ in $U_{0}$ and $i_{0} \geq 1$ such that for all $i \geq i_{0}, h^{-1} \mid V$ $\simeq f_{i}^{\prime} \mid V$ in $U_{0}, f_{i}\left|U \simeq f_{i+1}\right| U$ in $V$ and $f_{i}^{\prime} f_{i} \mid U \simeq 1_{U}$ in $U_{0}$. By 2.6 and 3.6 we can find an embedding $\hat{f}$ of $A$ into $V$ such that $\hat{f}(A)$ is a strong $Z_{n-k-2}$-set in $E^{n}$ and $\hat{f} \simeq f_{i_{0}} \mid A$ in $V$. Hence $\hat{f} \simeq f_{i} \mid A$ when $i \geq i_{0}$ and we have

$$
\begin{aligned}
h^{-1} \circ \hat{f} & \simeq h^{-1} \circ\left(f_{i} \mid A\right) \text { in } h^{-1}(V) \\
& =\left(h^{-1} \mid V\right) \circ\left(f_{i} \mid A\right) \simeq\left(f_{i}^{\prime} \mid V\right) \circ\left(f_{i} \mid A\right) \text { in } U_{0} \\
& =\left(f_{i}^{\prime} f_{i}\right) \mid A \simeq 1_{A} \quad \text { in } U_{0} .
\end{aligned}
$$

(2) Here and throughout $\S 4$, the notation " $a \simeq b$ in $T$ " means that the maps $a$ and $b$ are homotopic in the subspace $T$. 
Hence $\hat{f} \simeq h \mid A$ in $h\left(U_{0}\right)$. By Theorem 2.8 there is a homeomorphism $r$ of $E^{n}$ such that $r=1$ outside $h\left(U_{0}\right)$ and $\hat{f}=r h \mid A$. Let $q=r \circ h$. Then $q \mid A=\hat{f}$ and so $q$ has the required properties.

Lemma 4.2. Let $X$ and $Y$ be nonempty compact strong $Z_{n-k-2}$-sets in $E^{n}$ $(k \geq 0, n \geq 2 k+2)$ such that $\operatorname{Sh}(X)=\operatorname{Sh}(Y)$. Then there is a homeomorphism of pairs between $\left(E^{n} / X, \hat{X}\right)$ and $\left(E^{n} / Y, \hat{Y}\right)$.

Proof. Let $\left\{T_{i}\right\}$ and $\left\{W_{i}\right\}$ be basic systems of neighborhoods for $X$ and $Y$ respectively. Let $\left\{g_{i}, X, Y\right\}$ and $\left\{g_{i}^{\prime}, Y, X\right\}$ be homotopy inverse fundamental sequences (in $E^{n}$ ). Apply 4.1 with $A=X, B=Y, f_{i}=g_{i}, f_{i}^{\prime}=g_{i}^{\prime}, U_{0}=E^{n}, h=1$ and $V_{0}=W_{1}$ to obtain $q=p_{1}$, a homeomorphism of $E^{n}$ such that $X \subset p_{1}^{-1}\left(W_{1}\right)$ and $p_{1}\left|X \simeq g_{i}\right| X$ in $W_{1}$ for almost all $i$. Our final homeomorphism will equal $p_{1}$ on $E^{n}-p_{1}^{-1}\left(W_{1}\right)$. Let $S_{2}=T_{2} \cap p_{1}^{-1}\left(W_{1}\right)$. Next, apply 4.1 with $A=Y, B=X$, $f_{i}=g_{i}^{\prime}, f_{i}^{\prime}=g_{i}, U_{0}=W_{1}, h=p_{1}^{-1}$ and $V_{0}=S_{2}$ to obtain $q=p_{2}^{-1}$, a homeomorphism of $E^{n}$ such that $p_{2}=p_{1}$ on $E^{n}-p_{1}^{-1}\left(W_{1}\right), Y \subset p_{2}\left(S_{2}\right)$ and $p_{2}^{-1}\left|Y \simeq g_{i}^{\prime}\right| Y$ in $S_{2}$ for almost all $i$. Our final homeomorphism will equal $p_{2}$ on $E^{n}-S_{2}$. Let $S_{3}=W_{3} \cap p_{2}\left(S_{2}\right)$. Next, apply 4.1 with $A=X, B=Y, f_{i}=g_{i}, f_{i}^{\prime}=g_{i}^{\prime}, U_{0}=S_{2}$, $h=p_{2}$ and $V_{0}=S_{3}$ to obtain $q=p_{3}$ such that $p_{3}=p_{2}$ on $E^{n}-S_{2}, X \subset p_{3}^{-1}\left(S_{3}\right)$ and $p_{3}\left|X \simeq g_{i}\right| X$ in $S_{3}$ for almost all $i$. Continue in this way, defining a homeomorphism $p: E^{n}-X \rightarrow E^{n}-Y$ by $p=p_{2 i}$ on $E^{n}-S_{2 i}$. Since $S_{2 i} \subset T_{i}$, $\left\{S_{2 i}\right\}$ is a basic system of neighborhoods of $X$, and since $p_{2 i}\left(S_{2 i}\right) \subset S_{2 i-1} \subset W_{2 i-1}$, $\left\{p_{2 i}\left(S_{2 i}\right)\right\}$ is a basic system of neighborhoods of $Y$. Thus $p$ extends to a homeomorphism of pairs between $\left(E^{n} / X, \hat{X}\right)$ and $\left(E^{n} / Y, \hat{Y}\right)$.

Before proving Lemma 4.3, we give some definitions. For $i=0$ or 1 let $A_{i}$ be a space with a base point $a_{i} \in A_{i}$. Letting $j=1$ when $i=0$ and $j=0$ when $i=1$, we say $\left(A_{0}, A_{0}-a_{0}, a_{0}\right)$ and $\left(A_{1}, A_{1}-a_{1}, a_{1}\right)$ have the same homotopy type as triples if there are maps $f_{i}: A_{i} \rightarrow A_{j}$ and homotopies $F_{i}: A_{i} \times I \rightarrow A_{i}$ such that $F_{i}(\cdot, 0)=f_{j} \circ f_{i}, \quad F_{i}(\cdot, 1)=1_{A_{i}}, \quad F_{i}\left(\left\{a_{i}\right\} \times I\right)=\left\{a_{i}\right\}$ and $F_{i}\left(\left(A_{i}-\left\{a_{i}\right\}\right) \times I\right) \subset A_{i}$ - $\left\{a_{i}\right\} . f_{i}$ is a triple homotopy equivalence and $f_{j}$ is its triple homotopy inverse.

Lemma 4.3. Let $X$ and $Y$ be disjoint nonempty compact pseudo-polyhedral subsets of $E^{2 k+1} \subset E^{n}(n \geq 2 k+2)$ having dimension $\leq k$. Let $\left(E^{n} / X, E^{n} / X-\hat{X}, \hat{X}\right)$ and $\left(E^{n} / Y, E^{n} / Y-\hat{Y}, \hat{Y}\right)$ have the same homotopy type as triples. Then $\operatorname{Sh}(X)$ $=\operatorname{Sh}(Y)$.

Proof. Since $X$ is pseudo-polyhedral in $E^{2 k+1}$, there exist polyhedra $\left\{P_{i}\right\}$ and regular neighborhoods $\left\{N_{i}\right\}$ as in Definition 3.0, and we may assume (by choosing subsequences) that $N_{i+1} \subset$ int $N_{i}$ for all $i$. If $r_{i}: N_{i} \rightarrow P_{i}$ is (the final stage of) a PL strong deformation retraction, it follows that for all $j>i, r_{i} \mid N_{j} \simeq r_{j}$ in int $N_{i}$. By taking the cartesian product of each $N_{i}$ with a small PL ball, we can enlarge $N_{i}$ to be a regular neighborhood of $P_{i}$ in $E^{n}$, and $r_{i}$ can be extended to this enlarged neighborhood by the projection map of $E^{n}$ onto $E^{2 k+1}$. Abusing notation we call the new neighborhoods and retractions $N_{i}$ and $r_{i}$, and we observe 
$r_{i} \mid N_{j} \simeq r_{j}$ in (int $N_{i} \cap E^{2 k+1}$ ) for all $j>i$. Similarly, we let $\left\{P_{i}^{\prime}\right\},\left\{N_{i}^{\prime}\right\}$ and $\left\{r_{i}^{\prime}\right\}$ be the polyhedra, (enlarged) regular neighborhoods and (extended) retractions corresponding to $Y$. Since $X \cap Y=\varnothing$ we may assume $N_{1} \cap N_{1}^{\prime}=\varnothing$.

When $0 \leq t \leq 1$, define $F_{t}: E^{2 k+1} \rightarrow E^{n}$ by $F_{t}\left(x_{1}, \ldots, x_{2 k+1}, 0, \ldots, 0\right)$ $=\left(x_{1}, \ldots, x_{2 k+1}, t, 0, \ldots, 0\right)$. Clearly we may assume $F_{1 / i}\left(N_{i} \cap E^{2 k+1}\right) \subset N_{i}$ and $F_{1 / i}\left(N_{i}^{\prime} \cap E^{2 k+1}\right) \subset N_{i}^{\prime}$ for each $i$.

Let $\hat{h}: E^{n} / X \rightarrow E^{n} / Y$ be the triple homotopy equivalence given in the hypothesis, and let $\hat{h}^{\prime}$ be its triple homotopy inverse. Let $h: E^{n}-X \rightarrow E^{n}-Y$ and $h^{\prime}: E^{n}-Y \rightarrow E^{n}-X$ be the obvious induced maps. Because $h$ and $h^{\prime}$ come from $\hat{h}$ and $\hat{h}^{\prime}$, we can choose $n(i) \geq i$ and $n^{\prime}(i) \geq i$ to satisfy $h^{\prime} \circ h \mid\left(N_{n(i)}-X\right)$ $\simeq 1$ in $N_{i}-X, h \circ h^{\prime} \mid\left(N_{n^{\prime}(i)}^{\prime}-Y\right) \simeq 1$ in $N_{i}^{\prime}-Y, h\left(N_{n(i)}-X\right) \subset$ int $N_{i}^{\prime}$ and $h^{\prime}\left(N_{n^{\prime}(i)}^{\prime}-Y\right) \subset$ int $N_{i}$. We may assume $n(j) \geq n(i)$ and $n^{\prime}(j) \geq n^{\prime}(i)$ whenever $j>i$.

Now we define our fundamental sequences. Let $f_{1}: E^{n} \rightarrow E^{n}$ be any continuous map which agrees with $h \circ F_{1 / n(1)} \circ r_{n(1)}$ on $N_{n(1)} ; f_{1}$ maps $N_{n(1)}$ into int $N_{1}^{\prime}-Y$. Next we define $f_{2}: E^{n} \rightarrow E^{n}$ in three stages as follows: (i) $f_{2}$ agrees with $h \circ F_{1 / n(2)} \circ r_{n(2)}$ on $N_{n(2)}$ (and therefore $f_{2}$ maps $N_{n(2)}$ into int $N_{2}^{\prime}-Y$ ); (ii) since $f_{2}\left|N_{n(2)} \simeq f_{1}\right| N_{n(2)}$ in int $N_{1}^{\prime}-Y$ [proof: $r_{n(1)} \mid N_{n(2)} \simeq r_{n(2)}$ in (int $N_{n(1)}$ $\cap E^{2 k+1}$ ), hence $F_{1 / n(2)} \circ\left(r_{n(1)} \mid N_{n(2)}\right) \simeq F_{1 / n(2)} \circ r_{n(2)}$ in (int $N_{n(1)}-E^{2 k+1}$ ), hence $F_{1 / n(1)} \circ r_{n(1)} \mid N_{n(2)} \simeq F_{1 / n(2)} \circ r_{n(2)}$ in (int $N_{n(1)}-E^{2 k+1}$ ), and the claim follows by applying $h$ to each side], the Borsuk extension theorem allows us to extend $f_{2}$ to $N_{n(1)}$ so that $f_{2}\left|N_{n(1)} \simeq f_{1}\right| N_{n(1)}$ in int $N_{1}^{\prime}-Y$; (iii) $f_{2}$ is extended to the rest of $E^{n}$ in an arbitrary fashion. By repeating this procedure, we can define inductively $f_{i}: E^{n} \rightarrow E^{n}$ to agree with $h \circ F_{1 / n(i)} \circ r_{n(i)}$ on $N_{n(i)}$ (it maps $N_{n(i)}$ into int $N_{i}^{\prime}-Y$ ) and such that if $1 \leq j<i, f_{i}\left|N_{n(j)} \simeq f_{j}\right| N_{n(j)}$ in (int $N_{j}^{\prime}-Y$ ). Similarly, interchanging primed and unprimed symbols, we define maps $f_{i}^{\prime}: E^{n} \rightarrow E^{n}$. The definitions make $f=\left\{f_{i}, X, Y\right\}$ and $f^{\prime}=\left\{f_{i}^{\prime}, Y, X\right\}$ fundamental sequences.

To prove $f^{\prime} \circ f$ is (fundamentally) homotopic to the identity sequence of $X$, we need only show that for any $i$, and for any $j \geq n\left(n^{\prime}(i)\right), f_{j}^{\prime} \circ f_{j} \mid N_{j} \simeq 1_{N_{j}}$ in int $N_{i}$. Let $Q_{j}=F_{1 / j} r_{j}\left(N_{j}\right)$. Then $Q_{j}$ is a subpolyhedron of $N_{j}$ having dimension $\leq k$, and $Q_{j} \cap X=\varnothing . h\left(Q_{j}\right)$ lies in (int $\left.N_{n^{\prime}(i)}^{\prime}-Y\right)$. Let $e_{j}: Q_{j} \rightarrow$ int $N_{n^{\prime}(i)}^{\prime}-Y$ be a PL map homotopic in (int $N_{n^{\prime}(i)}^{\prime}-Y$ ) to $h \mid Q_{j}$. Clearly $e_{j}$ is PL homotopic in int $N_{n^{\prime}(i)}^{\prime}$ to $F_{1 / n^{\prime}(i)} \circ r_{n^{\prime}(i)}^{\prime} \circ e_{j}$ (which also maps $Q_{j}$ into int $N_{n^{\prime}(i)}^{\prime}-Y$ ). Since the image of this PL homotopy has dimension $\leq k+1 \leq n-k-1$, Proposition 3.2 allows us to move the homotopy off $Y$ and to assert $e_{j} \simeq F_{1 / n^{\prime}(i)} \circ r_{n^{\prime}(i)}^{\prime} \circ e_{j}$ in (int $N_{n^{\prime}(i)}^{\prime}-Y$ ). Hence $h\left|Q_{j} \simeq F_{1 / n^{\prime}(i)} \circ r_{n^{\prime}(i)}^{\prime} \circ h\right| Q_{j}$ in (int $N_{n^{\prime}(i)}^{\prime}-Y$ ). Applying $h^{\prime}$ to each side, replacing $Q_{j}$ by $F_{1 / j} r_{j}\left(N_{j}\right)$, and using the homotopies $F_{1 / j} \circ r_{j} \simeq 1_{N_{j}}$ in $N_{j}$, and $h^{\prime} \circ h \mid N_{j} \simeq 1_{N_{j}}$ in int $N_{i}$, we find $1_{N_{j}} \simeq f_{n^{\prime}(i)}^{\prime} \circ f_{j} \mid N_{j}$ in int $N_{i}$. Since $f_{n^{\prime}(i)}^{\prime}\left|N_{n^{\prime}(i)}^{\prime} \simeq f_{j}^{\prime}\right| N_{n^{\prime}(i)}^{\prime}$ in int $N_{i}$, we conclude $f_{j}^{\prime} \circ f_{j} \mid N_{j} \simeq 1_{N_{j}}$ in int $N_{i}$ as was to be shown. Similarly $f \circ f^{\prime}$ is fundamentally homotopic to the identity sequence of $Y$. 
Lemma 4.4. Let $X$ and $Y$ be compacta, let $x$ be a discrete point of $X=X^{\prime} \cup\{x\}$, let $y$ be a discrete point of $Y=Y^{\prime} \cup\{y\}$, and let $\operatorname{Sh}(X)=\operatorname{Sh}(Y)$. Then $\operatorname{Sh}\left(X^{\prime}\right)$ $=\operatorname{Sh}\left(Y^{\prime}\right)$.

Proof. Let $f$ and $\underline{g}$ be mutually homotopy inverse fundamental sequences between $X$ and $Y$. By Lemma 10.1 of [3] there is a bijection between the components of $X$ and the components of $Y$ such that corresponding components have the same shape: let $X_{1}$ correspond to $\{y\}$ and let $Y_{1}$ correspond to $\{x\}$. Then $\operatorname{Sh}\left(X_{1}\right)=\operatorname{Sh}\left(Y_{1}\right)=\operatorname{Sh}$ (point). The proof of Lemma 10.1 of [3] shows more: namely, that the fundamental equivalences between corresponding components are all obtained by suitably restricting $f$ and $\underline{g}$. This is easily seen to imply that $X-X_{1}$ and $Y-Y_{1}$ are compact. Therefore, by suitably restricting $f$ and $g$ we have $\operatorname{Sh}\left(X-\left(X_{1} \cup\{x\}\right)\right)=\operatorname{Sh}\left(Y-\left(Y_{1} \cup\{y\}\right)\right)$. If $X_{1}=\{x\}$ then $Y_{1}=\{y\}$ and we are done. If $X_{1} \neq\{x\}$ then $X^{\prime}$ [resp. $Y^{\prime}$ ] is the union of the two disjoint compacta $X-\left(X_{1} \cup\{x\}\right)$ and $X_{1}$ [resp. $Y-\left(Y_{1} \cup\{y\}\right)$ and $Y_{1}$ ]. Clearly this implies $\operatorname{Sh}\left(X^{\prime}\right)=\operatorname{Sh}\left(Y^{\prime}\right)$.

Proof of Theorem 1.1. (1) $\Rightarrow(2)$ is Lemma 4.2. (2) $\Rightarrow(3)$ is trivial. (2) $\Rightarrow$ (4) is trivial.

Proof of $(3) \Rightarrow(1)$. By 2.3, $X$ and $Y$ have dimension $\leq k$. Hence Theorem 3.3 allows us to find disjoint pseudo-polyhedral sets $X^{\prime}$ and $Y^{\prime}$ lying in $E^{2 k+1} \subset E^{n}$, such that $X$ is homeomorphic to $X^{\prime}$ and $Y$ is homeomorphic to $Y^{\prime}$. By Proposition 3.2, $X^{\prime}$ and $Y^{\prime}$ are strong $Z_{n-k-2}$-sets in $E^{n}$. By Theorem 2.8 , there is a homeomorphism of $E^{n}$ taking $X$ onto $X^{\prime}$, and another taking $Y$ onto $Y^{\prime}$. Hence $\left(E^{n} / X^{\prime}, E^{n} / X^{\prime}-\hat{X}^{\prime}, \hat{X}^{\prime}\right)$ and $\left(E^{n} / Y^{\prime}, E^{n} / Y^{\prime}-\hat{Y}^{\prime}, \hat{Y}^{\prime}\right)$ have the same homotopy type as triples. Lemma 4.3 implies $\operatorname{Sh}\left(X^{\prime}\right)=\operatorname{Sh}\left(Y^{\prime}\right)$ and hence $\operatorname{Sh}(X)=\operatorname{Sh}(Y)$.

Proof of (4) $\Rightarrow(1)$. Let $S=E^{n} \cup\{z\}$ be the one point compactification of $E^{n}$, and let $z^{\prime} \in S-(X \cup Y \cup\{z\})$. By hypothesis there is a homeomorphism $h: S-(X \cup\{z\}) \rightarrow S-(Y \cup\{z\})$ and we can arrange that $h\left(z^{\prime}\right)=z^{\prime}$. Let $h^{\prime}: S-\left\{z^{\prime}\right\} \rightarrow E^{n}$ be a homeomorphism, let $X^{\prime}=h^{\prime}(X \cup\{z\})$ and let $Y^{\prime}$ $=h^{\prime}(Y \cup\{z\})$. Then $h^{\prime} \circ h \circ\left(h^{\prime}\right)^{-1}$ induces a pair-homeomorphism between $\left(E^{n} / X^{\prime}, \hat{X}^{\prime}\right)$ and $\left(E^{n} / Y^{\prime}, \hat{Y}^{\prime}\right)$. Since $(2) \Rightarrow(3) \Rightarrow(1)$, we conclude $\operatorname{Sh}\left(X^{\prime}\right)$ $=\operatorname{Sh}\left(Y^{\prime}\right)$. Lemma 4.4 then implies $\operatorname{Sh}(X)=\operatorname{Sh}(Y)$.

Proof of Theorem 1.2. In case of hypothesis (a), let $k=\max (\operatorname{dim} X, \operatorname{dim} Y)$. We may assume $n-k-2 \geq 1$ : this only fails if $n=2$ and $k=0$, but then $E^{n}-X$ or $E^{n}-Y$ fails to be $1-$ ULC. Now combine 2.2 with 1.1. In case of hypothesis (b), combine 2.4 with 1.1 .

\section{REFERENCES}

1. R. D. Anderson, On topological infinite deficiency, Michigan Math. J. 14 (1967), 365-383. MR 35 \#4893.

2. R. H. Bing and J. M. Kister, Taming complexes in hyperplanes, Duke Math. J. 31 (1964), 491-511. MR 29 \#1626.

3. K. Borsuk, Concerning homotopy properties of compacta, Fund. Math. 62 (1968), 223-254. MR 37 \#4811. 
4. K. Borsuk, Remark on a theorem of S. Mardešić, Bull. Acad. Polon. Sci. Sér. Math. Astronom. Phys. 19 (1971), 475-483.

5. J. L. Bryant, On embeddings of compacta in Euclidean space, Proc. Amer. Math. Soc. 23 (1969), 46-51. MR 39 \# 6286.

6. - On embeddings of 1-dimensional compacta in $E^{5}$, Duke Math. J. 38 (1971), 265-270. MR 43 \# 1154.

7. J. L. Bryant and C. L. Seebeck III, An equivalence theorem for embeddings of compact absolute neighborhood retracts, Proc. Amer. Math. Soc. 20 (1969), 256-258. MR 39 \#6285.

8. T. A. Chapman, On some applications of infinite-dimensional manifolds to the theory of shape, Fund. Math. 76 (1972), 181-193.

9. - Shapes of finite-dimensional compacta, Fund. Math. 76 (1972), 261-276.

10. R. Geoghegan and R. R. Summerhill, Infinite-dimensional methods in finite-dimensional geometric topology, Bull. Amer. Math. Soc. 78 (1972), 1009-1014.

11. H. Gluck, Embeddings in the trivial range, Ann. of Math. (2) 81 (1965), 195-210. MR 30 \#3456.

12. D. Henderson, Applications of infinite-dimensional manifolds to quotient spaces of complete ANR's, Bull. Acad. Polon. Sci. Sér. Sci. Math. Astronom. Phys. (to appear).

13. J. F. P. Hudson, Piecewise linear topology, Benjamin, New York, 1969. MR 40 \# 2094.

14. W. Hurewicz and H. Wallman, Dimension theory, Princeton Math. Ser., vol. 4, Princeton Univ. Press, Princeton, N. J., 1941. MR 3, 312.

15. D. R. McMillan, Taming Cantor sets in $E^{n}$, Bull. Amer. Math. Soc. 70 (1964), 706-708. MR 29 \#1628.

16. M.A. Štan'ko, The embedding of compacta in Euclidean space, Mat. Sb. 83(125) (1970), 234-255. = Math USSR Sb. 12 (1970), 234-255. MR 42 \#6804.

17. $\mathrm{H}$. Bothe, $A$ tangled 1-dimensional continuum in $E^{3}$ which has the cube-with-handles property, Bull. Acad. Polon. Sci. Sér. Sci. Math. Astronom. Phys. 20 (1972), 481-486.

School of Mathematics, The Institute for Advanced Study, Princeton, New Jersey 08540

Current address (Ross Geoghegan): Department of Mathematics, State University of New York, Binghamton, New York 13901

Current address(R. Richard Summerhill): Department of Mathematics, Kansas State University, Manhattan, Kansas 66502 